\title{
Toughness and Longest Cycles in 2-Connected Planar Graphs
}

\section{T. Böhme*}

INSTITUT FÜR MATHEMATIK, TU ILMENAU PF 327, 98684 ILMENAU, GERMANY

\author{
H. J. Broersma \\ H. J. Veldman \\ FACULTY OF APPLIED MATHEMATICS \\ UNIVERSITY OF TWENTE \\ P.O. BOX 217, 7500 AE ENSCHEDE \\ THE NETHERLANDS \\ E-mail:broersma@math.utwente.nI
}

\begin{abstract}
Let $G$ be a planar graph on $n$ vertices, let $c(G)$ denote the length of a longest cycle of $G$, and let $\omega(G)$ denote the number of components of $G$. By a well-known theorem of Tutte, $c(G)=n$ (i.e., $G$ is hamiltonian) if $G$ is 4-connected. Recently, Jackson and Wormald showed that $c(G) \geq \beta n^{\alpha}$ for some positive constants $\beta$ and $\alpha \approx 0.2$ if $G$ is 3 -connected. Now let $G$ have connectivity 2 . Then $c(G)$ may be as small as 4 , as with $K_{2, n-2}$, unless we bound $\omega(G-S)$ for every subset $S$ of $V(G)$ with $|S|=2$. Define $\xi(G)$ as the maximum of $\omega(G-S)$ taken over all 2-element subsets $S \subseteq V(G)$. We give an asymptotically sharp lower bound for the toughness of $G$ in terms of $\xi(G)$, and we show that $c(G) \geq \theta \ln n$ for some positive constant $\theta$ depending only on $\xi(G)$. In the proof we use a recent result of Gao and Yu improving Jackson and Wormald's result. Examples show that the lower bound on $c(G)$ is essentially best-possible. (c) $1996 \mathrm{John}$ Wiley \& Sons, Inc.
\end{abstract}

\footnotetext{
* This research was carried out while the first author was visiting the Faculty of Applied Mathematics, University of Twente, supported by the Euler Institute for Discrete Mathematics and its Applications.
}

Journal of Graph Theory Vol. 23, No. 3, 257-263 (1996)

(c) 1996 John Wiley \& Sons, Inc. 


\section{INTRODUCTION}

We use [1] for basic terminology and notation not defined here, and consider finite simple graphs only. The circumference $c(G)$ of a graph $G$ is the length of a longest cycle in $G$. Suppose that $G$ is a planar graph on $n$ vertices. It is well-known from a theorem of Tutte [8] that $c(G)=n$ (i.e., $G$ is hamiltonian) if $G$ is 4-connected. Recently it has been shown that $c(G) \geq \beta n^{\alpha}$ for some positive constants $\beta$ and $\alpha$ if $G$ is 3-connected [5], [7]. If $G$ is 2 -connected but not 3-connected, its circumference may be as small as 4 , as with $K_{2, n-2}$. In rough terms, the absence of long cycles in $K_{2, n-2}$ is due to the fact that it contains two vertices the deletion of which results in a graph with many components. If the number of components one can get by deleting two vertices is bounded by a constant, the situation changes drastically.

Let $G$ be a connected graph. A subset $S$ of the vertex set $V(G)$ of $G$ is a separator of $G$ if the graph $G-S$, obtained from $G$ by deleting the vertices of $S$, is disconnected. A separator $S$ of $G$ is said to be a $k$-separator if $|S|=k$. An edge $x y$ of a 2-connected graph $G$ is said to be a good edge if $\{x, y\}$ is not a separator of $G$. If $G$ is a graph, then $\omega(G)$ denotes the number of components of $G$. For a 2-connected graph $G$ we define $\xi(G)$ to be the smallest natural $k$ such that for any 2-element subset $S$ of $V(G), \omega(G-S) \leq k$. For convenience we say that a cycle $C$ of a graph $G$ is an $X$-cycle if $E(C)$ contains the edges of $X \subseteq E(G)$.

Our main theorem reads as follows.

(1.1) Let $G$ be a 2-connected planar graph on $n$ vertices with $\xi(G) \geq 2$ and $f$ a good edge of $G$. Then $G$ has an $\{f\}$-cycle of length at least

$$
\gamma\left(\frac{1}{\xi(G)-1}\right)^{0.4} \ln n
$$

where $\gamma \approx 0.10$.

The proof of (1.1) is postponed until Section 3.

The parameter $\xi(G)$ is closely related to the concept of toughness in graphs which has been introduced by Chvátal [3] as follows. A connected graph $G$ is said to be $t$-tough if $|S| \geq t \cdot \omega(G-S)$ for every separator $S$ of $G$. The toughness $\tau(G)$ of a connected non-complete graph $G$ is defined by

$$
\tau(G)=\min \frac{|S|}{\omega(G-S)}
$$

where the minimum is taken over all separators $S$ of $G$. It is easy to see that every hamiltonian graph is 1-tough. The converse is not true, even in the case of maximal planar graphs (see [6]).

If $G$ is a 2 -connected graph with $\xi(G) \geq 2$, then, obviously,

$$
\tau(G) \leq \frac{2}{\xi(G)} .
$$

Consequently, (1.1) implies that every planar 2-connected graph $G$ on $n$ vertices with $\xi(G) \geq 2$ has a cycle of length at least $\gamma(\tau(G) /(2-\tau(G)))^{0.4} \ln n$. In general, there is no non-trivial lower bound for the toughness in terms of $\xi(G)$, but for planar graphs there is one. 
(1.2) Let $G$ be a 2-connected planar graph and $S$ a separator of $G$ such that $\tau(G)=$ $|S| / \omega(G-S)$. Then $\tau(G)=2 / \xi(G)$ if $|S|=2$, and $\tau(G)>1 /(3(\xi(G)-1)+2)$ otherwise.

The proof of (1.2) will be given in Section 3; moreover, examples will be provided showing that the bound is asymptotically sharp.

Although there is a long-standing conjecture stating that every 2-tough graph is hamiltonian (see e.g. [3]), there are only a few results concerning bounds for the circumference of $t$-tough graphs. The following theorem in [2] seems to be the best result for the case of 2-connected but not 3-connected graphs known up to now.

(1.3) (a) Let $t>0$ be fixed. If $G$ is a $t$-tough 2-connected graph on $n$ vertices, then

$$
c(G) \cdot \ln c(G) \geq 2 \ln n-o(\ln n) \quad(n \rightarrow \infty) .
$$

(b) For any real number $t, 0<t \leq 1$, and for any integer $n \geq 3$, there exists a $t$-tough 2-connected graph $G$ on $n$ vertices such that

$$
c(G)< \begin{cases}\frac{2}{\ln \left[\frac{1}{t}\right]} \cdot \ln n+4 & \text { if } 0<t \leq \frac{1}{2} \\ 4 \log _{2}(n+2)-4 \log _{2} 3+4 & \text { if } \frac{1}{2}<t \leq 1\end{cases}
$$

The proof of (1.3)(b) is constructive and all constructed graphs are planar. Thus the function $\ln n$ in the bound of (1.1) cannot be replaced by a function growing faster than $\ln n$.

\section{PRELIMINARIES}

A plane graph is a planar graph with a specified embedding in the euclidean plane. If $G$ is a plane graph and $C$ a cycle of $G$, then the induced embedding of $C$ is a simple closed curve in the plane. Thus, if $x$ is a vertex or an edge of $G$ but not of $C$, then $x$ is either inside or outside $C$. It is a well-known fact that each face of a 2-connected plane graph is bounded by a cycle. A cycle of a plane graph is called a facial cycle if it bounds a face.

Let $G$ be a 2-connected plane graph and $C$ a facial cycle of $G$. Then the ordered pair $(G, C)$ is said to be a circuit graph if for any 2-separator $S$ of $G$ every component of $G-S$ contains a vertex of $V(C)$. The proof of the following statement is an easy exercise, and is therefore omitted.

(2.1) Let $G$ be a 3-connected plane graph and $C$ a cycle of $G$. Furthermore, let $H$ be the plane graph obtained from $G$ by deleting all vertices and edges inside $C$. Then $(H, C)$ is a circuit graph.

Note that in (2.1) "inside $C$ " may be replaced by "outside $C$ ".

In [5] the following theorem is proved.

(2.2) There exists a positive constant $\beta \approx 0.193 \ldots$ such that for every circuit graph $(G, C)$ on $n$ vertices and every two edges $f, g \in E(C)$ there is an $\{f, g\}$-cycle of $G$ of length at least $\beta n^{0.4}+2$.

Combining (2.1) and (2.2), we get the following. 
(2.3) Let $G$ be a 3-connected plane graph on $n$ vertices and $f, g$ two edges of $G$. Then there is an $\{f, g\}$-cycle of $G$ of length at least

$$
\beta\left(\frac{n}{2}\right)^{0.4}+2
$$

where $\beta$ is the same constant as in (2.2).

Proof. Since $G$ is 3-connected there is a cycle $C$ of $G$ containing $f, g$. Let $G_{1}$ and $G_{2}$ denote the plane graphs obtained from $G$ by deleting all vertices and edges inside $C$ or outside $C$, respectively. Clearly, $\left|V\left(G_{1}\right)\right|+\left|V\left(G_{2}\right)\right|>n$. Hence, we may assume without loss of generality that $\left|V\left(G_{1}\right)\right|>\frac{1}{2} n$. Since (2.1) implies that $\left(G_{1}, C\right)$ is a circuit graph, the result now follows from (2.2).

Let $G$ be a graph, $f=x y$ an edge of $G$ and $P$ a path with endvertices $x^{\prime}, y^{\prime}$ such that $V(G) \cap V(P)=\emptyset$. Then the graph $H$ defined by $V(H)=V(G) \cup V(P)$ and $E(H)=$ $E(G) \cup E(P) \cup\left\{x x^{\prime}, y y^{\prime}\right\} \backslash\{x y\}$ is said to be obtained from $G$ by replacing $f$ by $P$. A graph $G^{\prime}$ is a subdivision of a graph $G$ if $G^{\prime}$ can be obtained from $G$ by replacing edges by paths. A superblock is either a complete graph on three vertices or a 3-connected graph. Let $H$ be a subgraph of a graph $G$ and $x$ a vertex of $V(G) \backslash V(H)$. Then $H$ is attached to $x$ if, in $G, x$ is adjacent to some vertex of $H$.

The following result plays a key role in our proof of (1.1).

(2.4) Let $G$ be a 2-connected graph and $f=x y$ an edge of $G$. Then there is a superblock $H$ and a subdivision $H^{\prime}$ of $H$ subject to the following conditions.

(1) $x y \in E(H) \cap E\left(H^{\prime}\right)$

(2) $H^{\prime}$ is a subgraph of $G$

(3) every component of $G-V(H)$ is attached to precisely two vertices of $V(H)$

(4) if $\{a, b\} \subseteq V(H)$ is a 2-separator of $G$, then $a b \in E(H)$.

Proof (by induction on $|V(G)|$ ). If $G$ has less than four vertices or $G$ is a superblock, then there is nothing to prove. Thus we may proceed with the inductive step and assume that $G$ has at least four vertices and contains a 2 -separator $S=\{u, v\}$. Let $G_{1}, \ldots, G_{k}$ be the components of $G-S$. We assume without loss of generality that $f$ is an edge of the subgraph of $G$ induced by $V\left(G_{1}\right) \cup S$. Now let $\tilde{G}$ be the graph obtained from $G$ by deleting $V\left(G_{2}\right)$ and adding the edge $u v$ unless $u v \in E(G)$. Obviously, $\tilde{G}$ is a 2-connected graph with fewer vertices than $G$ and $f$ is an edge of $\tilde{G}$. By the induction hypothesis there is a superblock $\tilde{H}$ and a subdivision $\tilde{H}^{\prime}$ of $\tilde{H}$ subject to (1), .., (4) with respect to $\tilde{G}, \tilde{H}$ and $\tilde{H}^{\prime}$ instead of $G, H$ and $H^{\prime}$. If $u v \in E(\tilde{H})$ or $\{u, v\} \nsubseteq V(\tilde{H})$, then we put $H=\tilde{H}$. Otherwise, $H$ is obtained from $\tilde{H}$ by adding the edge $u v$. If $u v \in E\left(\tilde{H}^{\prime}\right) \backslash E(G)$ or $u v \in E(H) \backslash E(\tilde{H})$, then $H^{\prime}$ is obtained from $\tilde{H}^{\prime}$ by adding a $(u, v)$-path in the subgraph of $G$ induced by $V\left(G_{2}\right) \cup S$ and deleting $u v$ provided $u v \in E\left(\tilde{H}^{\prime}\right)$. Otherwise, we let $H^{\prime}=\tilde{H}^{\prime}$. Now it is not hard to prove that $H^{\prime}$ is a subdivision of $H$ and that $G, H$, and $H^{\prime}$ satisfy the conditions (1), ., (4).

(2.5) Let $x$ be a real number with $x>1$. Then

$$
\frac{x^{0.4}}{\ln x} \geq \frac{2 e}{5} .
$$


Proof. Let $f(x)=x^{0.4} / \ln x$. For $x>1, f(x)$ has a continuous derivative $f^{\prime}(x)=$ $0.4 /\left(x^{0.6} \cdot \ln x\right)-1 /\left(x^{0.6} \cdot(\ln x)^{2}\right)$. Now it is easy to see that $x_{0}=e^{2.5}$ is the only $x>1$ for which $f^{\prime}(x)=0$. Since $f^{\prime}(x)<0$ for $1<x<x_{0}$ and $f^{\prime}(x)>0$ for $x>x_{0}, f\left(x_{0}\right)=2 e / 5$ is the unique minimum of $f(x)$ for $x>1$.

\section{PROOFS}

\subsection{Proof of (1.1)}

Instead of (1.1) we shall prove the following slightly modified statement.

(3.1) Let $G$ be a 2-connected planar graph on $n$ vertices, $f$ a good edge of $G$ and $\gamma=$ $\beta / 6^{0.4} \cdot 2 e / 5$, where $\beta$ is the same as in (2.2). Then $G$ has an $\{f\}$-cycle of length at least $l$, where

$$
l= \begin{cases}\gamma \ln n & \text { if } \xi(G)=1 \\ \gamma\left(\frac{1}{\xi(G)-1}\right)^{0.4} \ln n & \text { otherwise }\end{cases}
$$

Proof. The proof is by induction on $n$. If $n<4$, then $G$ is a complete graph on three vertices which is a (hamiltonian) cycle. Thus we may proceed with the inductive step and assume $n \geq 4$. By (2.4) there is a superblock $H$ containing $f$, and a subdivision $H^{\prime}$ of $H$ subject to the conditions (1), .., (4) of (2.4). We distinguish two cases.

Case 1. $V(G)=V(H)$. Then $G$ is a superblock and hence $G$ is 3-connected and $\xi(G)=1$. By (2.2) there exists an $\{f\}$-cycle of $G$ of length at least $\beta \cdot n^{0.4}$. Now it follows from (2.5) that

$$
\beta \cdot n^{0.4} \geq \gamma \ln n
$$

Case 2. $V(G) \neq V(H)$. Then by (3) every component of $G-V(H)$ is attached to precisely two vertices of $V(H)$. Let $n_{H}$ denote the number of vertices of $H$. Obviously, $H$ is a planar graph and, therefore, it follows from (4) and Euler's formula that the total number of components of $G-V(H)$ is at most $(\xi(G)-1)\left(3 n_{H}-6\right)$. Consequently, there is a component $F$ of $G-V(H)$ containing at least

$$
\frac{n-n_{H}}{(\xi(G)-1)\left(3 n_{H}-6\right)}
$$

vertices. Let $F$ be attached to $a, b \in V(H)$. Let $F^{\prime}$ be the subgraph of $G$ induced by $V(F) \cup\{a, b\}$, with the edge $a b$ added unless $a b \in E(G) . F^{\prime}$ is a 2-connected planar graph and $a b$ a good edge of $F^{\prime}$. Clearly, $\xi\left(F^{\prime}\right) \leq \xi(G)$. By the induction hypothesis there is an $\{a b\}$-cycle $C_{1}$ in $F^{\prime}$ of length at least

$$
\gamma\left(\frac{1}{\xi(G)-1}\right)^{0.4} \ln \left(\frac{n-n_{H}}{(\xi(G)-1)\left(3 n_{H}-6\right)}+2\right) .
$$

By (4), $H$ contains the edge $a b$. Consequently, it follows from (2.3) that there is an $\{f, a b\}$ cycle $C^{\prime}$ in $H$ of length at least $\beta\left(n_{H} / 2\right)^{0.4}+2$ if $H$ is 3-connected; the same is true if $H=K_{3}$. Since $G$ contains a subdivision $H^{\prime}$ of $H$, there is a cycle $C^{\prime \prime}$ in $H^{\prime}$ which is a 
subdivision of $C^{\prime}$. If $a b \in E\left(C^{\prime \prime}\right)$, let $C_{2}=C^{\prime \prime}$; otherwise denote by $P$ the path $a b$ has been replaced with, and let $C_{2}$ be the cycle obtained from $C^{\prime \prime}$ by deleting the inner vertices of $P$ and adding $a b$. Clearly $C_{2}$ is an $\{f, a b\}$-cycle containing no vertices of $F$ which is at least as long as $C^{\prime}$. Consequently, the subgraph of $G$ obtained from $C_{1} \cup C_{2}$ by deleting the edge $a b$ is an $\{f\}$-cycle of length at least

$$
\beta\left(\frac{n_{H}}{2}\right)^{0.4}+2+\gamma\left(\frac{1}{\xi(G)-1}\right)^{0.4} \ln \left(\frac{n-n_{H}}{(\xi(G)-1)\left(3 n_{H}-6\right)}+2\right)-2 .
$$

Since $\xi(G) \geq 2\left(\right.$ and $\left.n_{H} \geq 0\right)$,

$$
\begin{aligned}
& \beta\left(\frac{n_{H}}{2}\right)^{0.4}+2+\gamma\left(\frac{1}{\xi(G)-1}\right)^{0.4} \ln \left(\frac{n-n_{H}}{(\xi(G)-1)\left(3 n_{H}-6\right)}+2\right)-2 \\
& \quad \geq \beta\left(\frac{n_{H}}{2}\right)^{0.4}+\gamma\left(\frac{1}{\xi(G)-1}\right)^{0.4} \ln n-\gamma\left(\frac{1}{\xi(G)-1}\right)^{0.4} \ln \left((\xi(G)-1) 3 n_{H}\right) .
\end{aligned}
$$

Consequently, it suffices to prove that

$$
\gamma \frac{1}{(\xi(G)-1)^{0.4}} \leq \frac{\beta\left(\frac{n_{H}}{2}\right)^{0.4}}{\ln \left((\xi(G)-1) 3 n_{H}\right)} .
$$

Let $x=(\xi(G)-1) 3 n_{H}$. Then

$$
\frac{\beta\left(\frac{n_{H}}{2}\right)^{0.4}}{\ln \left((\xi(G)-1) 3 n_{H}\right)}=\frac{\beta}{6^{0.4}(\xi(G)-1)^{0.4}} \cdot \frac{x^{0.4}}{\ln x}
$$

and the result follows from (2.5) and the definition of $\gamma$.

\subsection{Proof of (1.2)}

Let $G$ be a planar 2-connected graph and $S \subseteq V(G)$ a $k$-separator of $G$ such that $\tau(G)=$ $|S| / \omega(G-S)$. If $k=2$, then $\omega(G-S)=\xi(G)$. Otherwise, it follows from Euler's formula that the number of components of $G-S$ attached to precisely two vertices of $S$ is at most $(\xi(G)-1)(3 k-6)$. In order to count the components of $G-S$ attached to more than two vertices of $S$, we consider the graph $G^{\prime}$ obtained from $G$ by deleting all edges of $G[S]$ and all components of $G-S$ which are attached to precisely two vertices of $S$ and by contracting all the other components of $G-S$ into single vertices, called the white vertices of $G^{\prime}$. Let $w$ denote the number of white vertices of $G^{\prime}$. Clearly, $G^{\prime}$ is planar and bipartite, so

$$
3 w \leq\left|E\left(G^{\prime}\right)\right| \leq 2\left|V\left(G^{\prime}\right)\right|-4=2(k+w)-4,
$$

implying that $w \leq 2 k-4$. Thus the total number of components of $G-S$ is $\xi(G)$ if $k=2$ and at most $2 k-4+(\xi(G)-1)(3 k-6)$ otherwise. Now the result follows directly from the definition of $\tau(G)$.

The following examples show that the bound on the total number of components of $G-S$ obtained in the above proof is sharp. Let $k \geq 3$ and $l \geq 1$ be fixed integers. Let $T_{k}$ be a plane triangulation on $k$ vertices. (A plane triangulation is a plane graph 
every face of which is bounded by a cycle of length three.) Clearly, $T_{k}$ has $3 k-6$ edges and $2 k-4$ faces. Now a new plane graph $G_{k}$ is constructed as follows. First, a new vertex $x_{f}$ is inserted in every face $f$ of $T_{k}$ such that $x_{f}$ is adjacent to the vertices on the boundary of $f$. Next, if $l \geq 2$, every edge of $T_{k}$ is replaced by $l-1$ parallel edges, each of which is then subdivided at least once. Now it is not hard to see that $\xi\left(G_{k}\right)=l, \tau\left(G_{k}\right)=$ $\left|V\left(T_{k}\right)\right| / \omega\left(G_{k}-V\left(T_{k}\right)\right)$, and $\omega\left(G_{k}-V\left(T_{k}\right)\right)=2 k-4+(l-1)(3 k-6)$. Consequently, $\tau\left(G_{k}\right)=k /(2 k-4+(l-1)(3 k-6))$, and $\lim _{k \rightarrow \infty} \tau\left(G_{k}\right)=1 /(3(l-1)+2)$.

\section{CONCLUDING REMARKS}

Using a proof technique similar to that in the proof of (1.2), for an $r$-connected planar graph $G(r \geq 3)$ one easily shows that $\omega(G-S) \leq(2|S|-4) /(r-2)$ for every separator $S$ of $G$. This implies that $\tau(G)>\frac{1}{2}(r-2)$ for such a graph $G$, and that $\omega(G-S) \leq|S|-2$ for every separator $S$ of a 4-connected planar graph $G$. The latter result recently appeared in [4].

Using (3.1) it is not difficult to show that through any (not necessarily good) edge $f=x y$ of a 2-connected planar graph $G$ on $n$ vertices there is a cycle of length at least $\gamma^{\prime} \ln n$ for some positive constant $\gamma^{\prime}$ depending on $\xi(G)$ only: simply use that $\omega(G-\{x, y\}) \leq \xi(G)$ and apply (3.1) to a suitable subgraph of $G$.

In [2] it is conjectured that any 2-connected $t$-tough graph on $n$ vertices contains a cycle of length at least $\zeta \ln n$, where $\zeta$ is a positive constant depending on $t$ only. As shown in [2], the conjecture is true for 3-connected graphs. Clearly our results imply the truth of the statement for planar graphs. We remark that the general conjecture could be proved along the lines of our proof of (1.1) if one could prove that every 3-connected $t$-tough graph $G$ on $n$ vertices contains an $\{f, g\}$-cycle of length at least $\zeta^{\prime} \ln n$ for any $f, g \in E(G)$ and some positive constant $\zeta^{\prime}$ depending only on $t$.

\section{References}

[1] J. A. Bondy and U. S. R. Murty, Graph Theory with Applications, Macmillan, London and Elsevier, New York (1976).

[2] H. J. Broersma, J. van den Heuvel, H. A. Jung, and H. J. Veldman, Long paths and cycles in tough graphs, Graphs and Combinatorics 9 (1993), 3-17.

[3] V. Chvátal, Tough graphs and hamiltonian circuits, Discrete Math. 5 (1973), 215-228.

[4] M. B. Dillencourt, On the toughness index of planar graphs, J. Graph Theory 18 (1994), 103107.

[5] Zhicheng Gao and Xingxing Yu, Convex programming and circumference of 3-connected graphs of low genus, preprint (1995).

[6] J. Harant and P. Owens, Non-hamiltonian 5/4-tough maximal planar graphs, Discrete Math. 147 (1995), 301-305.

[7] B. Jackson and N. C. Wormald, Longest cycles in 3-connected planar graphs, J. Combin. Theory Ser. B 54 (1992), 291-321.

[8] W. T. Tutte, A theorem on planar graphs, Trans. Amer. Math. Soc. 82 (1956), 99-116.

Received October 18, 1995 